\title{
Design and Implementation of Artwork Display System Based on Internet of Things Technology
}

\author{
Qian Zhao ${ }^{1,2}$ and Chenglin $\mathrm{Wu}$ (iD ${ }^{3}$ \\ ${ }^{1}$ Guangzhou Academy of Fine Arts, Guangzhou, 510006 Guangdong, China \\ ${ }^{2}$ Graduate Institute of Creative Industries, College of Management, Shih Chien University, Taipei 10001, Taiwan \\ ${ }^{3}$ School of Computer Science, Huanggang Normal College, Huanggang, 438000 Hubei, China
}

Correspondence should be addressed to Chenglin Wu; wuchenglin@hgnu.edu.cn

Received 15 November 2021; Accepted 18 January 2022; Published 27 February 2022

Academic Editor: Nima Jafari Navimipour

Copyright (C) 2022 Qian Zhao and Chenglin Wu. This is an open access article distributed under the Creative Commons Attribution License, which permits unrestricted use, distribution, and reproduction in any medium, provided the original work is properly cited.

\begin{abstract}
The IoT brings digital and intelligent changes, which can change many ways of seeing the world. Among them, the display of rare works of art is easily overlooked. This paper studies the artwork display system based on the IoT technology and uses the Spring framework under the uRLLC communication protocol to design the artwork display system through the design of the database and the controller. This article takes the artistic work display system as the research object, studies the operating mode of the system model, conducts system testing in an indoor environment, and compares communication with traditional distributed computing technology. Based on the experimental results, it can be seen that the real-time performance of this system is far superior to traditional cloud computing systems.
\end{abstract}

\section{Introduction}

The Internet of Things (IoT) has been applied in many fields. In the field of visualization, there are often higher standards for the reliability and real-time performance of IoT devices. In addition, as there are more and more IoT devices, and these devices are usually heterogeneous, how to manage these devices will be an urgent problem to be solved. And edge computing provides a new way to solve these problems. Edge computing can realize on-site processing of IoT device data, provide the system with high realtime performance, and ensure the reliability of communication.

Zhu et al. applied augmented reality technology to the exhibition of cultural relics and developed a cultural relic display system that supports the interaction of flashlight light [1]. Levin et al. used augmented reality technology to achieve a restoration display of the frescoes in St. Clement's Church [2]. Chakraborty et al. combine the outdoor AR system with the Yuanmingyuan Ruins to realize the digital res- toration of ancient images such as water viewing method, east-west water tower, and big water method [3]. Taking the world cultural heritage Dujiangyan as an example, Zou et al. use digital technology to attract the audience to understand water conservancy knowledge and historical stories based on the characteristics of Dujiangyan's regional protection. In addition, digital technology is used to improve the existing wasteland in the Dujiangyan Scenic Area and promote Dujiangyan tourism [4]. Zou et al. used AR technology to carry out a digital display and experience project of material cultural heritage ARCHEOGUIDE on mobile devices, with the purpose of recreating cultural heritage scenes for tourists through visual display methods [5]. Wei et al. use digital animation, virtual reality, and other technologies to digitally display representative European culture and superimpose virtual information on the cultural relics of the ancient city through AR technology [6]. Jian et al. elaborated on the protection methods of cultural heritage in the new era and proposed that the combination of cultural heritage and AR will become the inevitable trend of cultural heritage protection [7]. 
Li uses AR technology to visualize the three-dimensional model of outdoor cultural heritage sites and provides users with cultural heritage information through the object panoramic visualization system [8]. Xuanyuan et al. use augmented reality technology to develop the Byblos system, through which users can visit the image structure of the Roman theater to understand the cultural background [9]. Based on mobile augmented reality technology (Mobile Augmented Reality, MAR for short) combined with the digital protection of intangible cultural heritage taking Longquan celadon as an example, Riliang et al. have researched and designed a system applied on a smartphone platform [10]. Sinha et al. use AR technology and somatosensory interaction technology to study how national orchestral instruments can perform virtual performance. His research makes people enjoy the fun of virtual combination when enjoying musical instruments. This application has successfully attracted people's interest in national musical instruments [11].

This article introduces the system architecture, database, edge computing server, and data processing flow are designed and explained in detail, and edge computing is combined with the display of artworks to provide users with smart electricity usage. In the future, we will continue to observe the stability of the system, improve the system's ability to process concurrency, and lay a firm foundation for building edge computing IoT systems.

\section{IoT Technology and Art Exhibition Architecture}

2.1. Hierarchical Architecture of Art Display System Based on AIoT Technology. With the further integration and application of AIoT technology in smart images, especially in its artwork display system, "artificial intelligence" has gradually developed to "applied intelligence," and smart images with image energy efficiency management and smart IoT applications as the core are especially developed. Green smart images have quietly become the development trend of future images, so it is very important to actively construct and carry out theoretical and applied research on "AIoT+smart images" [12], in order to improve the feasibility and scalability of intelligent image artworks during system development and the safety and reliability of the system during actual operation [13]. On the basis of the original three-tier system architecture of the intelligent image art display system, combined with the actual fusion application of AIoT technology, the overall hierarchical structure of the intelligent image art display system based on AIoT is finally formed, that is, a total of 5 layers from bottom to top. The terminal equipment layer, the intelligent perception layer, the network communication layer, the AI cloud brain control layer, and the application scenario layer are composed. This system architecture can make the structure of the largest and most complex artwork display system in the intelligent image clearer and the division of labor. Among them, the terminal equipment layer is located at the bottom of the architecture and is the ultimate bearer of green smart images to achieve "green" and "intelligence," generally including building system smart terminals that implement various specific functions [14]. The intelligent perception layer refers to all kinds of intelligent devices with communication capabilities in the green intelligent image, as well as smart sensors and controllers, which can perform preliminary information processing and provide basic data for the application scenario layer and mainly carry the information transfer obtained from the perception layer. The AI cloud brain control layer is equivalent to an artificial intelligence control decisionmaking platform; that is, through AI intelligent units, intelligent algorithms and information technology to complete the mining, calculation, analysis, statistics, classification, and integration of real-time data and information of each subsystem can finally be achieved. The autonomous learning and control, intelligent decision-making and organization, and personalized customization are the functions used; the application scenario layer is to establish a system integration platform for each module system of specific users according to the different requirements of different users [15].

In recent years, with the continuous deepening of the country's new urbanization process, the country, provinces, prefectures, cities, and related industries have successively issued "green policies," related industry standards and regulations, which have strongly promoted the construction and development of smart images. Globalization, especially the 30 years of digitalization in our country, is model era $\rightarrow \mathrm{PC}$ interconnection era $\rightarrow$ mobile internet $\mathrm{era} \rightarrow \mathrm{IoT} \rightarrow$ smart IoT evolving, especially with the rapid development and integration of AI technology and IoT technology. While information and data provide vitality to $\mathrm{AI}, \mathrm{AI}$ is also creating all possibilities for IoT. The AIoT technology has become an effective way and the best way to intelligently upgrade major traditional industries represented by the image industry in the next ten years. Smart image is an ideal combination of "AI+smart image." Its core is "artificial intelligence platform," that is, "AI cloud brain." The overall architecture of the system composed of intelligent image "AI cloud brain" is composed of perception layer, transmission layer, and control layer. It has the characteristics of high precision, low cost, complete functions, and strong automation [16]. The transport layer generally refers to a comprehensive technical system composed of $5 \mathrm{G}$ and AI computing; the control layer is mainly composed of clusters such as control, decision-making, execution, and management. The Chinese name of "AIoT" is intelligent IoT, that is, "AI+IoT," which is based on the organic combination, effective combination, and application integration of artificial intelligence and IoT [17]. In fact, the concept of AIoT was proposed as early as 2017 and was first popularized by the industry. In recent years, it has become a hot vocabulary and industry hotspot in the IoT industry and the image industry [18].

2.2. NB-IoT Technology. The smart art display system based on NB-IoT uses the following technologies: sensor technology, IoT wireless remote communication technology, and cloud platform technology, so that terminal managers can remotely monitor the temperature and humidity data of the art display system and take corresponding measures [19]. This system is composed of temperature and humidity 
sensor module, GPS module, NB-IoT module, and transparent cloud platform module. The temperature and humidity module adopts the industrial-grade temperature and humidity sensor SHT20, and the GPS module model adopts the L70-R which supports low power consumption mode [20]. The temperature and humidity sensor collects the temperature and humidity of the indoor system environment, and the GPS sensor collects the geographic location [21]. The visual engineering measurement platform based on IoT technology and cloud computing combines the characteristics of engineering measurement and is divided into three levels: data acquisition layer, data analysis and storage layer, and management and application layer on the basis of IoT application mode [22].

2.2.1. Data Collection Layer. The data collection layer covers the perception layer and network layer of the IoT system architecture and is responsible for collecting measurement data and uploading the data to the designated server [23]. The IoT is responsible for connecting numerous devices, computers, and built-in sensors in a dynamic network and continuously receiving and exchanging information from the external environment [24]. In engineering surveys, the total station and GPS-RTK-integrated machine can complete most of the survey tasks, so the platform is mainly designed for the data networking of these two types of equipment [25]. The GPS-RTK-integrated machine adopts relative positioning measurement method to eliminate errors such as ephemeris, ionosphere, and troposphere; combined with carrier phase measurement technology, cmlevel high-precision measurement can be achieved, which can meet the requirements of engineering measurement. The data transmission uses the 5G IoT module. Compared with the traditional radio transmission method, the data transmission method of the $5 \mathrm{G}$ IoT module is not easily affected by the surrounding environment and transmission power, and the transmission distance is not limited. The measurement data can be directly uploaded to the server. Relative positioning measurement requires the use of two satellite receivers, a base station and a mobile station. The base station is placed in a location with stable geological conditions and known three-dimensional coordinates. During the measurement, the base station collects satellite observation information and sends the observation data and its own three-dimensional coordinates to the mobile station through the $5 \mathrm{G}$ IoT module. While the measuring station obtains the measurement information sent from the base station, it also collects the satellite observation information, and the information is calculated in real time, and then, the calculated data with a positioning accuracy of $\mathrm{cm}$ level is obtained. Finally, the $5 \mathrm{G}$ IoT module is used to upload the calculated data to the server. Considering that the current smart phones are equipped with Bluetooth communication function, an Android-based URLLC communication terminal is designed, which uses the Bluetooth communication function of the mobile phone to establish a connection with the total station to realize wireless control of the total station and at the same time uses the mobile phone network communication function to communicate with the server to realize data transmission.

2.2.2. Data Analysis and Storage Layer. The data analysis and storage layer use cloud computing technology to analyze the raw data measured by the equipment and store the data for subsequent use.

2.2.3. Management and Application Layer. The management and application layer adopts the B/S architecture, that is, the general browser/server architecture on the Internet. The back end adopts Spring, SpringMVC, Mybatis, and other frameworks, and the front end adopts Vue, Axios, and Element-ui frameworks; the front and back ends use very lightweight and language-independent JSON for data interaction. The B/S architecture uses the HTTP protocol for communication, and business processing is performed on the server. There is no need to develop and maintain client programs, and cross-platform operations can be easily realized, ensuring the security of the platform and data to the greatest extent. In order to facilitate the internal collaborative measurement in engineering measurement, the platform is designed to implement a management platform and a measurement platform. The management platform runs in the browser on the PC side to provide personnel with functions such as data management, measurement task management, and report management; the measurement platform runs on the browser of the mobile device (smart phone); a visual measurement interface is provided for the measurement personnel to improve the efficiency of the measurement operation. The entire measurement platform application layer design is divided into management platform and measurement platform.

Considering that engineering measurement operations are mainly divided into known point stakeout and unknown point measurement, the platform designs a set of internal collaborative measurement operations process based on these two measurement operation methods and corresponding operation procedures, combined with the platform application mode. Create a measurement task on the management platform and assign it to the surveyor. The surveyor can select the available measurement equipment on the platform to start the visual measurement operation. After the measurement is completed, it will be submitted to the staff for review. The task that passes the review can be exported to the report, and the review is not passed. The task needs to be reassigned to the surveyor for measurement. Through the powerful storage and computing capabilities of the cloud computing center, the requirements of the system can be met.

2.3. SAS Logical Structure Model Based on Improved Hypergraph. "Super network" is used to describe a system with many nodes and nested networks in the network. Hypergraph theory is one of the methods with a relatively complete mathematical definition. A hypergraph is a twotuple $\mathrm{V}$ is a finite set of nodes, satisfying the formula:

$$
G=G_{w}+G_{n b}+G_{t}
$$




$$
G=\frac{\sum_{j=1}^{k} \sum_{h=1}^{k} \sum_{t=1}^{n_{j}} \sum_{r=1}^{n_{h}}\left|y_{i j}-y_{h r}\right|}{2 n^{2} u} .
$$

A hypergraph can be regarded as a generalization of graph theory. Its edges can include any number of nodes. The finite set of nodes with the same attributes is the hyperedge of the hypergraph. The definition of hypergraph ignores the connection relationship between nodes. However, whether in the definition of weights in hypernetworks or in the study of flows, the connection relationship between nodes is of great significance. Improve the definition of hypergraph by increasing the connection relationship between nodes, so that it includes both the definition of nodes and edges in basic graph theory and the definition of super edges in hypergraph theory:

$$
\begin{gathered}
u_{h} \leq u_{j} \leq \cdots \leq u_{k}, \\
G j j=\frac{1 / 2 u_{j} \sum_{i=1}^{n_{j}} \sum_{r=1}^{n_{j}}\left|y_{j i}-y_{j r}\right|}{n_{j}^{2}}, \\
G w=\sum_{j=1}^{k} G_{j j} p_{j} s_{j} .
\end{gathered}
$$

Combined with the introduction of the characteristics of commonly used centrality indicators in related literature, the actual T1-1 network is investigated, and the neighbor-based node centrality and the path-based node subgraph centrality are selected as indicators to evaluate the importance of nodes. At the same time, these two centrality indicators are easy to extend to the hypergraph, and the definition in the hypergraph is equally clear. Logical nodes are ranked according to relative (super) degree centrality, and the top three LNs are the same, namely, IHMI (human machine interaction), TCTR, and TVTR. In terms of (super) degree centrality, they are the most important, because they have more connections with other nodes, and they also appear more frequently in different functions:

$$
\begin{gathered}
G_{j h}=\frac{\sum_{Z=1}^{h_{j}} \sum_{r=1}^{n_{h}}\left|y_{j i}-y_{h r}\right|}{n_{j} n_{h}\left(u_{j}+u_{h}\right)}, \\
G_{n b}=\sum_{j=2}^{k} \sum_{h=1}^{j-1} G_{j h}\left(p_{j} s_{h}+p_{h} s_{j}\right) D_{j h}, \\
G_{t}=\sum_{j=2}^{k} \sum_{h=1}^{j-1} G_{j h}\left(p_{j} s_{h}+p_{h} s_{j}\right) D_{j h}\left(1-D_{j h}\right) .
\end{gathered}
$$

In the SAS-improved hypergraph modeling process, the logical nodes in the SAS logical structure are defined as the nodes of the SAS-improved hypergraph model, the logical connections between logical nodes are defined as edges, and the logical function composed of several logical nodes is defined as super side. Take the T1-1 type substation in IEC61850 as an example to carry out the improved hyper- graph modeling and related analysis of SAS. Each logical node (LN) is a subfunction located in a physical node, and only data in the LN can interact. There may be a logical connection between two LNs, and each functional super edge contains multiple LNs:

$$
\begin{gathered}
D_{j h}=\frac{d_{j h}-P_{j h}}{d_{j h}+P_{j h}}, \\
d_{j h}=\int_{0}^{\infty} d F_{j}(y) \int_{0}^{y}(y-x) d F_{h}(x), \\
d_{j h}=\int_{0}^{\infty} d F_{h}(y) \int_{0}^{y}(y-x) d F_{j}(y) .
\end{gathered}
$$

The improved hypergraph model of SAS logical structure is a triplet. The node set $\mathrm{V}$ in the triplet is combined with the edge set GE and the hyperedge set HGE, respectively, which can form the SAS graph model GG=VE and the hypergraph model $\mathrm{HG}=\mathrm{VE}$ :

$$
\begin{gathered}
f(x)=\frac{1}{N h} \sum_{i=1}^{N} k\left(\frac{X_{i}-x}{h}\right), \\
k(x)=\frac{1}{\sqrt{2 \pi}} \exp \left(-\frac{x^{2}}{2}\right), \\
h_{t}=\tanh \left(w_{c} x_{t}+u_{c}\left(r_{t} \Theta h_{t-1}\right)+b_{c}\right), \\
h_{t}=z_{t} \Theta h_{t-1}+\left(1-z_{t}\right) \Theta h_{t} .
\end{gathered}
$$

In the graph structure, the objects appearing in pairs are related to a certain degree. Among them, objects are abstracted into nodes in the graph, and the "association" between two objects is called an edge. Graph models have advantages when analyzing networks or complex networks. Based on the mathematical definition of the graph and some basic parameters used to describe the graph, some commonly used parameters to measure the topological structure characteristics of the graph model can be derived. The definitions of these parameters are as follows:

$$
\begin{gathered}
\sigma t=\frac{\sqrt{1 / n \sum_{i=1}^{n}\left(F I_{i t}-F I_{i t}\right)^{2}}}{F I_{i t}}, \\
u_{(j \mid i)}=w_{i j} A_{i}, \\
s_{j}=\sum_{i} c_{i j} u_{(j \mid i)} .
\end{gathered}
$$

Using the definitions and formulas of these parameters, the basic parameter values of the T1-1 SAS logic graph model can be calculated. The position of a node in the network topology determines its criticality and scope of influence. Structural centrality is the most commonly used indicator for evaluating the importance of nodes. They measure the criticality of nodes themselves by influencing the capabilities of other nodes. The neighbor-based centrality indicators and path-based centrality indicators are two 
commonly used centrality indicators:

$$
\begin{gathered}
\ln \left(\frac{F I_{i t}}{F I_{i t}-1}\right)=\alpha+\beta \ln F I_{i t}-1+v_{i}+\mathfrak{J}_{t}, \\
c_{i j}=\frac{e^{b_{i j}}}{\sum_{k} e^{b_{i k}}} .
\end{gathered}
$$

The degree centrality index is a typical neighbor-based centrality index, which is defined as the number of adjacent nodes of a node. According to the degree centrality index, the more direct neighbors of a node, the more critical it is. When considering the information dissemination process, when the path through the node can spread information faster and more widely, the node is more critical, and this can be calculated by the centrality index based on the path, and the centrality of the subgraph is a typical. The pathbased centrality index is as follows:

$$
\begin{gathered}
r=\frac{\alpha}{1-\beta}, \\
\theta=-\frac{1}{T} \ln (1+\beta), \\
\tau=\frac{\ln (2)}{\theta} .
\end{gathered}
$$

They are the most vulnerable nodes based on degree centrality. Once they fail, they can have a significant impact on SAS. For other LNs, the ranking of superdegree and degree is quite different. For example, ITCI is ranked fourth in the ranking of relative hyper centrality, but it is not yet ranked in the top ten in relative hyper centrality. This shows that there is a logical connection between ITCI and fewer LNs, but it appears in more super edges:

$$
\begin{gathered}
\ln \left(\frac{F I_{i t}}{F I_{i t}-1}\right)= \\
k_{t 1}[i]=\sum_{j} \cos \left(w_{i}^{1}, w_{j}^{2}\right) .
\end{gathered}
$$

Logical nodes are sorted according to relative (super) subgraph centrality. As shown in (25), the ordering of each LN according to relative subgraph centrality and relative hypersubgraph centrality is very different. PDIS has the greatest hypersubgraph centrality, which means that the closed loop of PDIS passes through more super edges and has more short closed paths than other LNs, and data deviations will propagate faster.

\section{Artwork Display System Design}

\subsection{System Hardware and Software Support}

3.1.1. Work of Art Data Analysis Server. The work of art data analysis service is based on the Spring framework, and the dependencies between objects are handed over to Spring to reduce the coupling between components; use nonblocking input/output (NIO) to solve high concurrency downloading artwork data transmission problem and improve server data processing capacity. The automated deployment of services is realized by writing shell scripts and combining with the maven packaging tool. The data analysis service is highly available and easy to expand. It can automatically handle client connections and deal with abnormal situations accordingly to ensure data security and accuracy.

3.1.2. uRLLC Communication Terminal. uRLLC communication terminal is based on the Android operating system and is responsible for data forwarding between the total station and the server. During the measurement, the server issues measurement instructions to the URLLC communication terminal, and the uRLLC communication terminal sends the received measurement instructions to the total station and obtains the measurement data returned by the total station and finally sends the measurement data to the server. In this process, the mobile phone acts as an intermediary for data transmission and is responsible for data forwarding, and the data processing will be carried out by the server.

3.1.3. Artwork Information Management Platform. The management platform is divided into 6 modules according to functions, and each module will carry out authority control to ensure the security of the platform and data. The user management module is responsible for the management of platform users and the corresponding role authority management. The equipment management module manages the communication parameters of the total station and the GPS-RTK-integrated machine and the server. The parameter management module is responsible for the basic parameters in engineering measurement such as projection parameters and coordinate systems. The basic control points and other parameters are stored and changed. The job management module is responsible for task creation, dispatch, audit, and report export. The operation log module records all logs of the platform to ensure that all user operations on the platform are traceable and commonly used the tool module provides common calculation tools such as coordinate conversion, seven-parameter calculation, fourparameter calculation, and geodetic calculation.

3.1.4. Visual Measurement Platform. The visual measurement platform is mainly divided into 3 modules. The surveyor can select the equipment being used in the equipment selection module, then select the task assigned to him in the construction measurement module, and enter the visualization interface for measurement; the custom module provides temporary operation functions. Surveyors can create tasks and complete measurements according to the on-site environment and assist or supplement the tasks assigned to them.

\subsection{System Design Steps}

3.2.1. Control Function. In order to complete the distributed access control decision, operations such as the decisionmaking authorization of the access control authority of the IoT device and the setting of the access control strategy 
and the management of user information are completed by four smart contracts deployed in the hyperledger, namely, the PIP contract, PAP contract, PDP contract, and PEP contract. In this section, we will introduce the contracts and functions that implement access control functions. In the PIP contract, the resource owner manages the access control authority of the IoT device and the identity of the registered user through this contract. The PIP contract contains the following functions:

(i) AddResource() function. This function is triggered when the resource owner uploads the access control authority of the IoT device

(ii) AddResource() function. This function writes the access control authority information of the device into the blockchain. A legal access control authority information requires four parameters

(iii) DeleteResource() function. Under certain circumstances, the resource owner no longer continues to share the access control authority of the device. Through this function, enter the resource ID to be revoked, and the system deletes the corresponding resource information in the blockchain to complete the resource cancellation; the resource applicant will not be able to search for information about the resource on the blockchain.

(iv) UserRegister() function. The user enters the user name and user ID in the user registration page; the system registers the user identity information through this function and saves it in the blockchain for subsequent system operations.

The PDP contract is responsible for judging whether the resource requester matches the access control policy of the applied device and judging whether the current resource requester is an authorized user of the device. The functions included in the contract are as follows: The MatchPolicy() function is mainly responsible for comparing the attribute information of the resource requester with the attribute information in the access control policy of the requested IoT device to determine whether it can obtain the access control authority of the device. This function is called by the GetPermission() function in the PEP contract when the resource requester applies for resource access control permissions.

The JudgeUser() function is responsible for judging whether the current resource requester is one of the authorized users of the device and passing the judgment result to the PEP contract. The judgment process is similar to the MatchPolicy() function, so I will not repeat it.

In the PEP contract, the contract is responsible for receiving the results returned by the PDP contract and executing corresponding actions based on the results. The PEP contract contains the following four functions:

(i) The ShowResource() function displays the obtained access control authority according to the entered user name (ii) The UseResource() function determines whether the device is included in the user's available resource list according to the result returned by the JudgeUser() function in the PDP contract

(iii) The FindResourceByRes_ID() function is called by the system when the resource requester searches for available equipment, and the corresponding search result is displayed according to the input resource ID

(iv) The GetPermission() function grants the access control permission of the device to the resource requester according to the result returned by the MatchPolicy() function in the PDP contract and uploads the permission information obtained by the resource requester to the blockchain.

3.2.2. Construction of BIM Core Database. The monitoring data is used for the construction of the BIM database, which mainly includes the data collection and transmission layer, the data processing and analysis layer, and the data storage layer. In the demonstration of real-time monitoring functions, the monitoring data and evaluation results need to be reflected in the BIM three-dimensional model for visual warning display, which is realized through the user interaction layer. The basis of monitoring visualization is the image model of the tunnel, including the positioning of the tunnel structure axis, the detailed structure, and the positioning of the sensors. In addition, the model and parameter information of equipment operation and maintenance and pipeline installation management are also applied to the BIM module, and the import interface is set to integrate the monitoring data in the database with the BIM 3D model. The technical realization is shown in Figure 1.

\section{Artwork Display System}

4.1. System Hardware Analysis. As shown in Figure 2, the landslide monitoring data collection system constructed by the wireless sensor network in this article is composed of two parts: a short-range wireless sensor network and a remote monitoring center. The wireless sensor network is the basis for building the IoT architecture, including sensor nodes, routing nodes, and assemble nodes, using the ZigBee protocol to build a clustered network structure, so as to realize mutual communication and information transfer between nodes.

As shown in Table 1, the choice of data transmission protocol determines the reliability and stability of data transmission in wireless sensor networks, compared with other transmission protocols such as Bluetooth, WiFi802.11, WirelessUSB, Bluetooth, Cellular, and ZigBee802.15.4. The transmission rate is low, but it can meet the small-capacity monitoring data transmission, the power consumption is low, the transmission distance can be expanded, and the overall implementation is easier.

The data transmission process is shown in Figure 3. Based on the IoT technology, this platform connects the GPS-RTK-integrated machine and the total station, two 


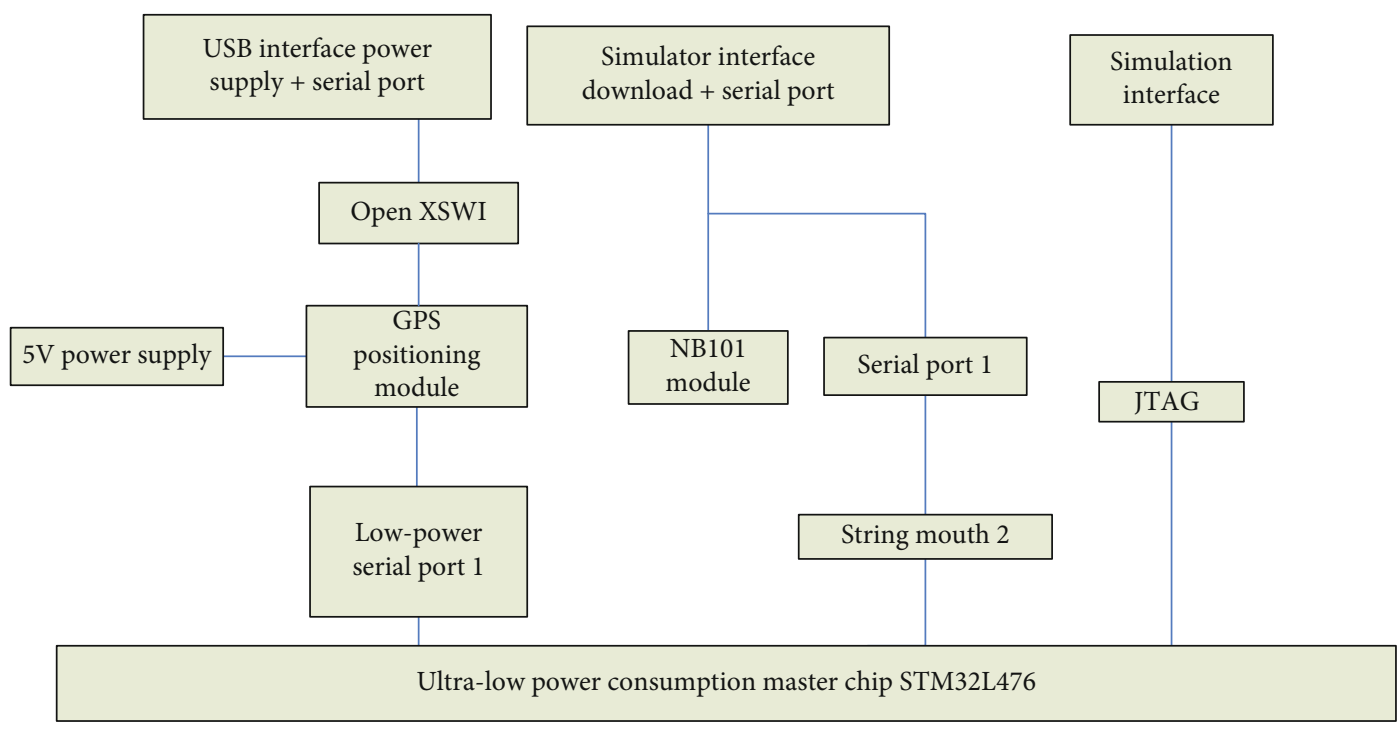

FIGURE 1: Integration of monitoring data in database with BIM 3D model.

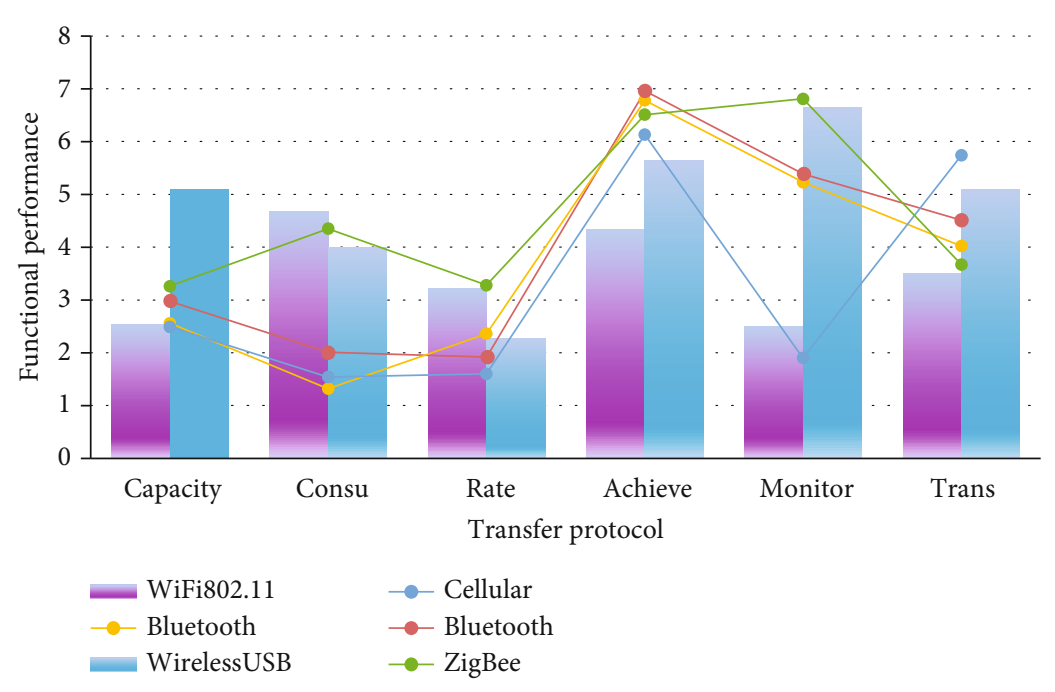

FIgURE 2: Reliability and stability of data transmission in the network.

TABLE 1: Reliability and stability of data transmission in wireless sensor networks.

\begin{tabular}{|c|c|c|c|c|c|c|}
\hline Item & Bluetooth & WiFi802.11 & WirelessUSB & Bluetooth & Cellular & ZigBee \\
\hline Capacity & 2.98 & 2.53 & 5.1 & 2.56 & 2.49 & 3.26 \\
\hline Consu & 2.01 & 4.67 & 4 & 1.32 & 1.54 & 4.35 \\
\hline Rate & 1.92 & 3.22 & 2.28 & 2.36 & 1.6 & 3.28 \\
\hline Achieve & 6.96 & 4.34 & 5.65 & 6.78 & 6.13 & 6.51 \\
\hline Monitor & 5.39 & 2.5 & 6.65 & 5.23 & 1.9 & 6.81 \\
\hline Trans & 4.51 & 3.51 & 5.1 & 4.02 & 5.74 & 3.67 \\
\hline
\end{tabular}

commonly used measurement equipment in engineering surveys, so that it can safely communicate with the server to achieve measurement data. At the same time, the platform is based on cloud computing technology and B/S architecture and has designed a management platform and measurement platform that can be operated across platforms. The stored measurement data is used to achieve internal collaborative measurement. The management platform provides unified management functions for engineering measurement data and work processes. The measurement platform realizes the visual measurement operation. The platform realizes the unification of online management and measurement, 


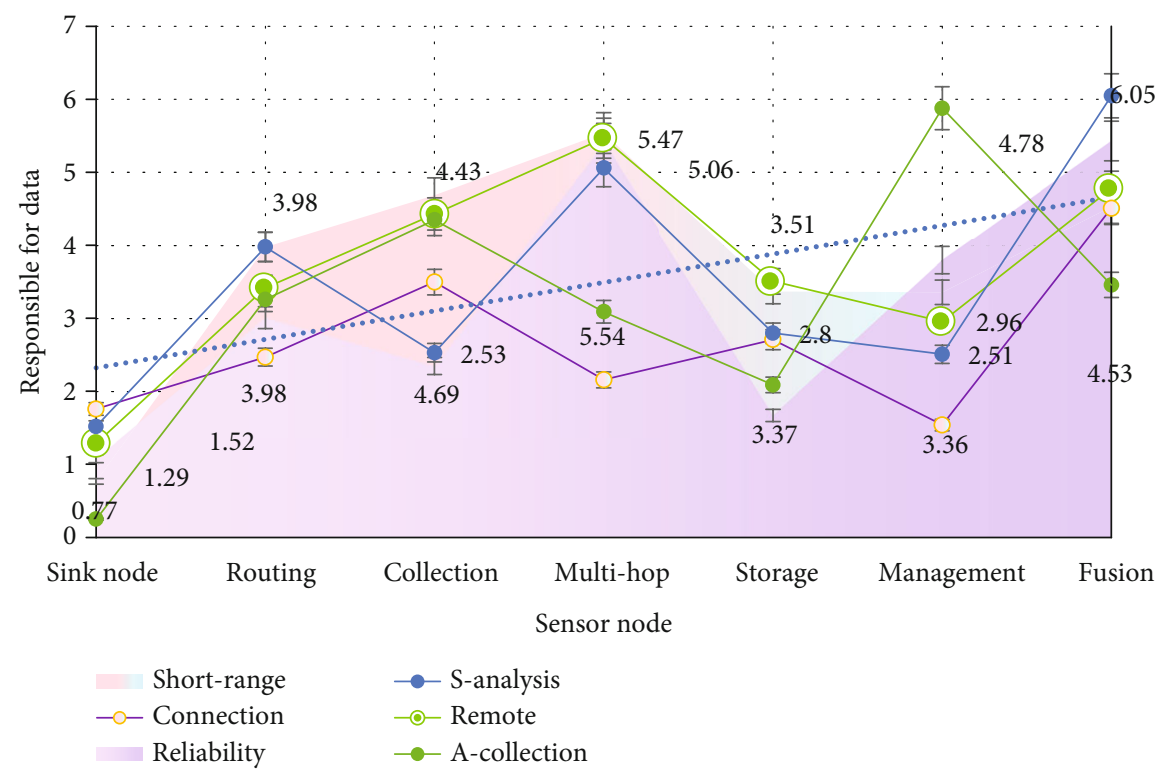

Figure 3: Artwork information data transmission process.

which can effectively improve the efficiency of engineering measurement and greatly reduce the overall cost of construction.

As shown in Table 2, this article mainly accesses two measuring devices, a total station and a GPS-RTKintegrated machine. When measuring with a total station device, it is highly dependent on the measurement function of the total station. What the platform does is to control the total station for measurement and then read and store the measurement results of the total station. The platform itself cannot improve the measurement accuracy of the total station. There are many factors affecting the measurement accuracy of GPS-RTK-integrated equipment. Therefore, the GPS-RTK all-in-one device was tested during the platform test, and the projection parameters are shown in Table 3.

As shown in Figure 4, the user authenticates his identity through the security authentication interface provided by the cloud computing layer and obtains a security token. The user uses the security token to issue control instructions to the edge layer through the device control channel of cloud computing and submit this control information to the system. If it is found through the network inspection that the user and the edge server are in the same local area network, the user is allowed to establish a secure connection with the edge server through a security token, and the user submits this control information to the system through the edge layer device. After receiving the control instruction, the edge layer device encapsulates the instruction through the conversion relationship and sends it to the target perception layer device. The sensing layer device executes the instruction and returns the result. The return process is consistent with the device upload process.

The selected plane coordinates of the known points are as follows: After creating the coordinate stakeout task, select the GPS-RTK all-in-one device for measurement, record 8 sets of measurement data, and obtain the measurement result graph, as shown in Figure 5. It is expressed in plane coordinates and normal height format. Compare the measured data in the figure with the original coordinates of the known points, and perform the difference calculation on $\mathrm{X}$, $\mathrm{Y}$, and $\mathrm{H}$ to obtain the deviation table, as shown in Table 4. The direction from a point on the ground to a point in the sky or another point on the ground is represented by the angle between the projection of the line connecting the two points on the horizontal plane and the reference direction. This representation is also called azimuth, which is one of the ways to measure the angular difference between objects on a plane. It is the horizontal included angle from the north direction line of a point to the target direction line in a clockwise direction. The analysis shows that when the GPS-RTK-integrated machine is used for measurement, it can ensure the measurement error within $1 \mathrm{~cm}$ in the horizontal direction and $2 \mathrm{~cm}$ in the elevation direction, which meets the engineering measurement requirements.

As shown in Figure 6, a monitoring terminal of this system is equipped with NB-IoT communication module, temperature and humidity sensor, photoresistor sensor, ethanol sensor, air quality sensor, etc. Turn on the power, the monitoring terminal will initialize the hardware, monitor the NB-IoT network attachment status, and access the cloud platform. Then, the monitoring terminal enters the normal environmental monitoring stage.

As shown in Table 5, in order to ensure the practicability of the system, this article tests the accuracy and completeness of its functions, including data collection, LCD display, abnormal alarm and remote data reporting, management, display, and other functions. The test is mainly through the indoor field test of the monitoring terminal and the remote test of the indoor monitoring management platform. The monitoring terminal is placed in the room for field test.

The LCD display screen of the monitoring terminal 1 is shown in Figure 7. This article separately displays the 
TABLE 2: Highly dependent on total station equipment measurement.

\begin{tabular}{|c|c|c|c|c|c|c|}
\hline Item & Short range & Remote & Reliability & Connection & S-analysis & A-collection \\
\hline Sink node & 0.77 & 1.29 & 1.08 & 1.76 & 1.52 & 0.25 \\
\hline Routing & 3.98 & 3.42 & 3.01 & 2.47 & 3.98 & 3.26 \\
\hline Collection & 4.69 & 4.43 & 2.35 & 3.5 & 2.53 & 4.35 \\
\hline Multihop & 5.54 & 5.47 & 5.4 & 2.16 & 5.06 & 3.09 \\
\hline Storage & 3.37 & 3.51 & 1.67 & 2.71 & 2.8 & 2.09 \\
\hline Management & 3.36 & 2.96 & 3.8 & 1.54 & 2.51 & 5.88 \\
\hline Fusion & 4.53 & 4.78 & 5.43 & 4.51 & 6.05 & 3.46 \\
\hline
\end{tabular}

TABle 3: Physical equipment test projection parameters.

\begin{tabular}{lcccccc}
\hline Item & Communication module & Data analysis & Packaging module & Data calculation & Response module & Data storage \\
\hline Edge & 4.23 & 3.34 & 2.86 & 3.93 & 3.67 & 2.59 \\
Storage & 2.31 & 3.91 & 3.18 & 2.92 & 1.43 & 4.28 \\
Traditional & 1.58 & 1.19 & 1.65 & 3.36 & 2.87 & 6.79 \\
Centralized & 4.48 & 4.52 & 1.67 & 2.22 & 6.15 & 5.77 \\
Cloud & 2.22 & 6.17 & 6.28 & 6.04 & 2.92 & 4.77 \\
Model & 5.34 & 6.09 & 4.89 & & & 2.41 \\
\hline
\end{tabular}

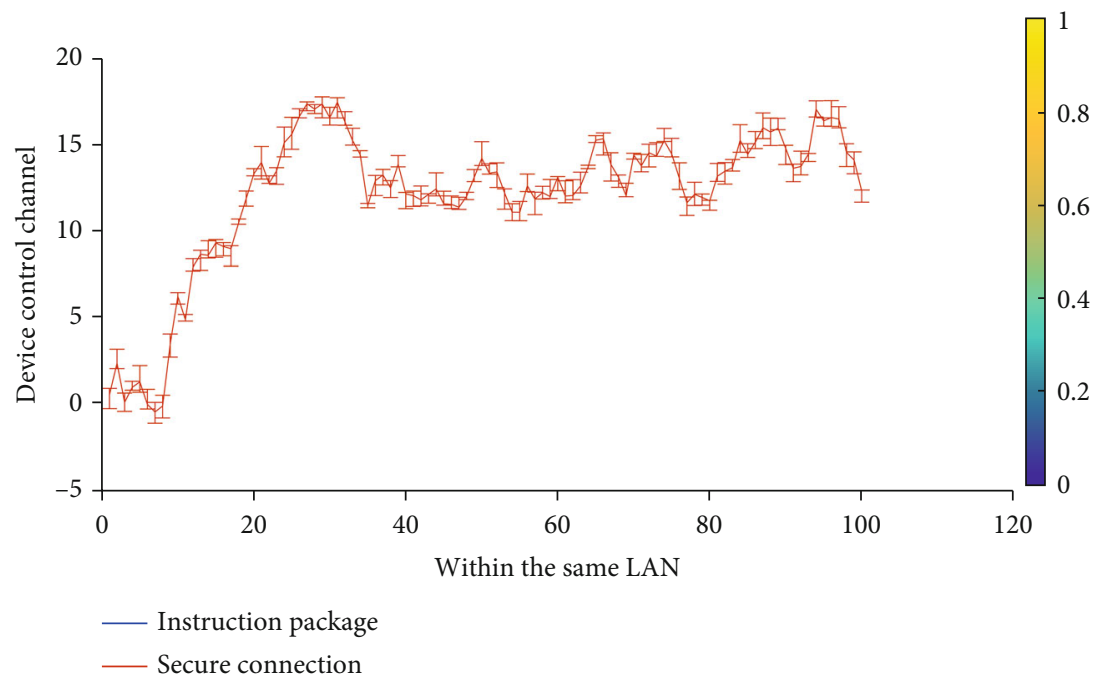

FIGURE 4: Security authentication interface authentication identity.

temperature, humidity, light intensity, ethanol concentration, air quality, voltage, and real-time time data collected by the monitoring terminal and their status. The normal temperature range is $10 \sim 16^{\circ} \mathrm{C}$, and the light intensity is normally less than $100 \mathrm{~lx}$, and the actual values are all outside the normal range. Therefore, the LCD displays that its status is abnormal, and the terminal activates a buzzer to alarm.

As shown in Figure 8, the indoor environment monitoring management platform built by the OneNET IoT cloud platform realizes the management of 30 monitoring terminals. At the same time, abnormal alarms can be viewed through the status display icon of each monitoring terminal.

At the same time, you can view the detailed data of each monitoring terminal through the view detail function. As shown in Table 6, the detailed data display interface of the monitoring terminal can be used to view the temperature, humidity, light intensity, ethanol concentration, air quality, and values such as voltage, and at the same time, you can view its changing trend through the graph. After the above tests, the monitoring terminal data collection, data display, abnormal alarm, and other functions are normal and meet the design requirements. At the same time, the interactive function of the monitoring terminal and the cloud platform is accurate and stable. The system completes one data collection, data display, abnormal judgment, and data reporting time, no more than 20 seconds, indicating that the functionality of the indoor environment monitoring system meets the expected requirements and is practical. 


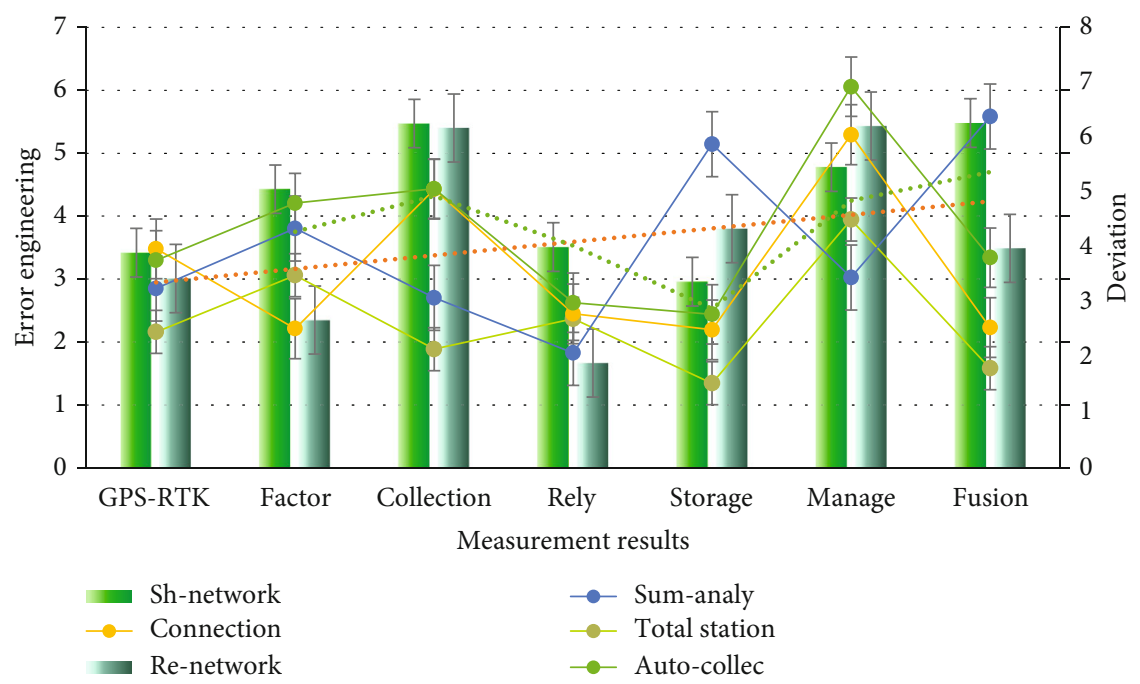

Figure 5: All-in-one machine 8 sets of data measurement results.

TABLE 4: Difference calculation comparison deviation.

\begin{tabular}{|c|c|c|c|c|c|c|}
\hline Item & Sh-network & Re-network & Total station & Connection & Sum-analy & Auto-collec \\
\hline GPS-RTK & 3.42 & 3.01 & 2.47 & 3.98 & 3.26 & 3.77 \\
\hline Factor & 4.43 & 2.35 & 3.5 & 2.53 & 4.35 & 4.81 \\
\hline Collection & 5.47 & 5.4 & 2.16 & 5.06 & 3.09 & 5.07 \\
\hline Rely & 3.51 & 1.67 & 2.71 & 2.8 & 2.09 & 3 \\
\hline Storage & 2.96 & 3.8 & 1.54 & 2.51 & 5.88 & 2.79 \\
\hline Management & 4.78 & 5.43 & 4.51 & 6.05 & 3.46 & 6.92 \\
\hline Fusion & 5.48 & 3.49 & 1.81 & 2.55 & 6.38 & 3.82 \\
\hline
\end{tabular}

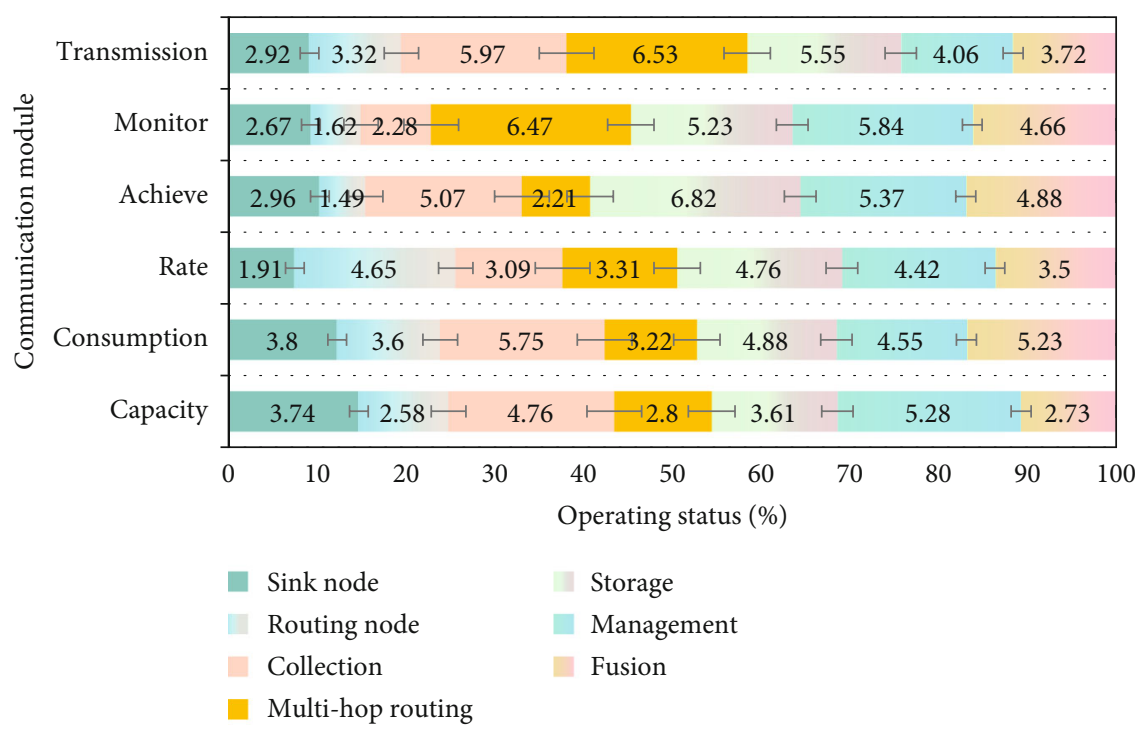

FIgURE 6: The monitoring terminal enters the normal environmental monitoring stage.

As shown in Figure 9, compared with the traditional centralized cloud computing model, this system adds an edge computing layer close to the sensing layer device and quickly processes the data of the sensing layer device through the computing and storage capabilities of the edge layer. The following article describes the design of the edge server. As shown in Table 7, the four functional modules of the edge server are as follows: communication module, data analysis and packaging module, data operation and response module, and data storage module. 
TABLe 5: Functional accuracy and completeness testing.

\begin{tabular}{|c|c|c|c|c|c|c|}
\hline & Sink node & Routing node & Collection & Storage & Management & Fusion \\
\hline Capacity & 3.74 & 2.58 & 4.76 & 3.61 & 5.28 & 2.73 \\
\hline Consumpt & 3.8 & 3.6 & 5.75 & 4.88 & 4.55 & 5.23 \\
\hline Rate & 1.91 & 4.65 & 3.09 & 4.76 & 4.42 & 3.5 \\
\hline Achieve & 2.96 & 1.49 & 5.07 & 6.82 & 5.37 & 4.88 \\
\hline Monitor & 2.67 & 1.62 & 2.28 & 5.23 & 5.84 & 4.66 \\
\hline Transmis & 2.92 & 3.32 & 5.97 & 5.55 & 4.06 & 3.72 \\
\hline
\end{tabular}

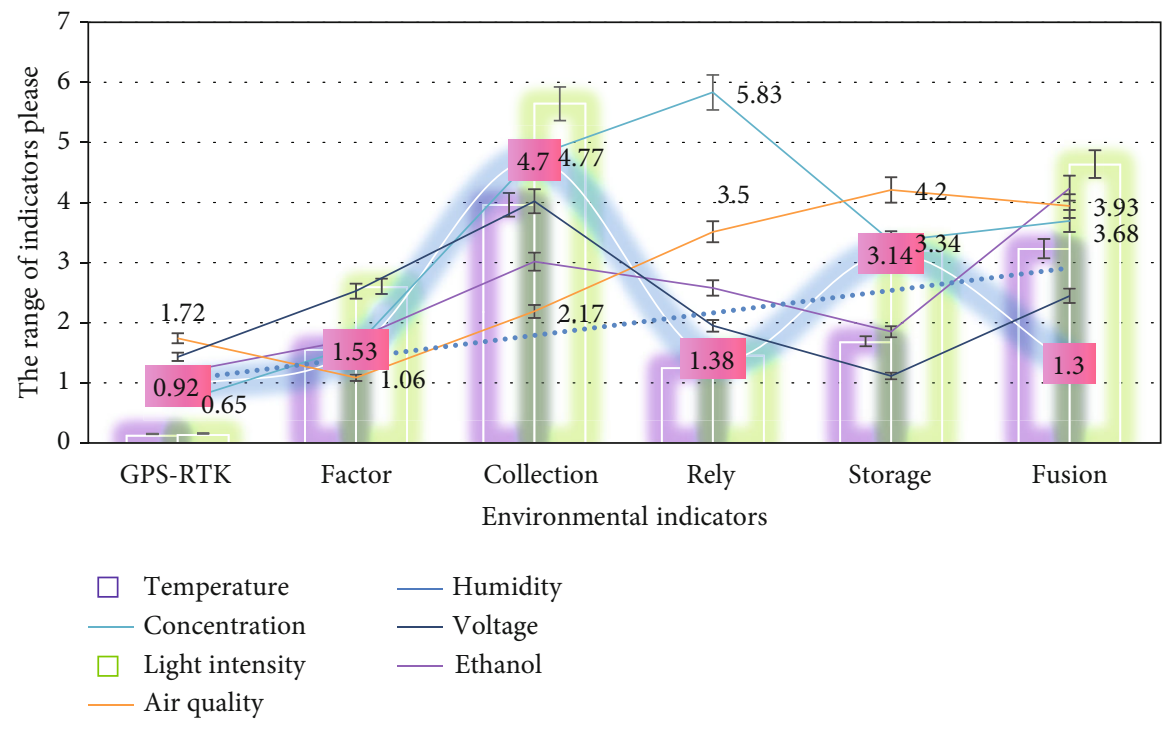

FIgURE 7: LCD display of monitoring terminal 1.

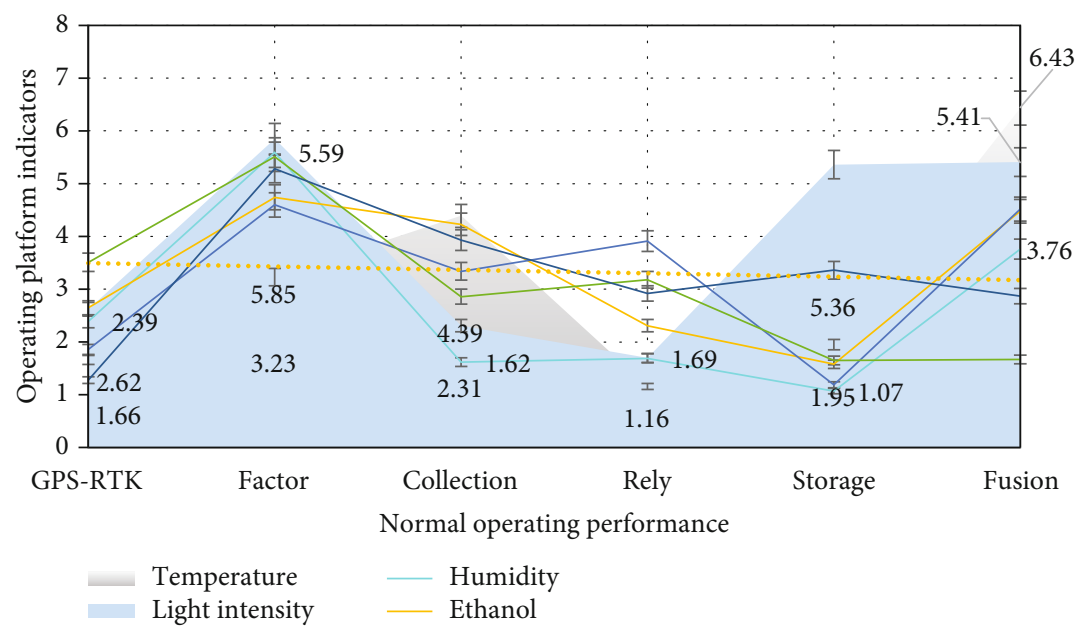

FIGURE 8: Remote monitoring indoor environment monitoring management platform.

As shown in Figure 10, through the communication layer, the system edge server can perceive the communication status of IoT devices and their communication status, obtain communication links, and provide access ports for new devices. At the same time, ensure smooth communication between the edge server and the cloud computing center, and ensure a smooth data flow channel. The equipment behavior in this system refers to the measurement and regulation of external monitoring variables and equipment variables.

As shown in Figure 11, when the sensing layer device generates data, it is uploaded to the edge layer device. The edge layer converts the data through the conversion relationship and, according to the data type, stores the data in the 
TABLE 6: Detailed data of each monitoring terminal.

\begin{tabular}{|c|c|c|c|c|c|c|c|}
\hline Item & Temperature & Humidity & Light intensity & Ethanol & Concentration & Air quality & Voltage \\
\hline GPS-RTK & 1.66 & 2.39 & 2.62 & 2.65 & 1.86 & 3.51 & 1.28 \\
\hline Factor & 3.23 & 5.59 & 5.85 & 4.74 & 4.6 & 5.51 & 5.28 \\
\hline Collection & 4.39 & 1.62 & 2.31 & 4.23 & 3.34 & 2.86 & 3.93 \\
\hline Rely & 1.16 & 1.69 & 1.7 & 2.31 & 3.91 & 3.18 & 2.92 \\
\hline Storage & 1.95 & 1.07 & 5.36 & 1.58 & 1.19 & 1.65 & 3.36 \\
\hline Fusion & 6.43 & 3.76 & 5.41 & 4.48 & 4.52 & 1.67 & 2.87 \\
\hline
\end{tabular}

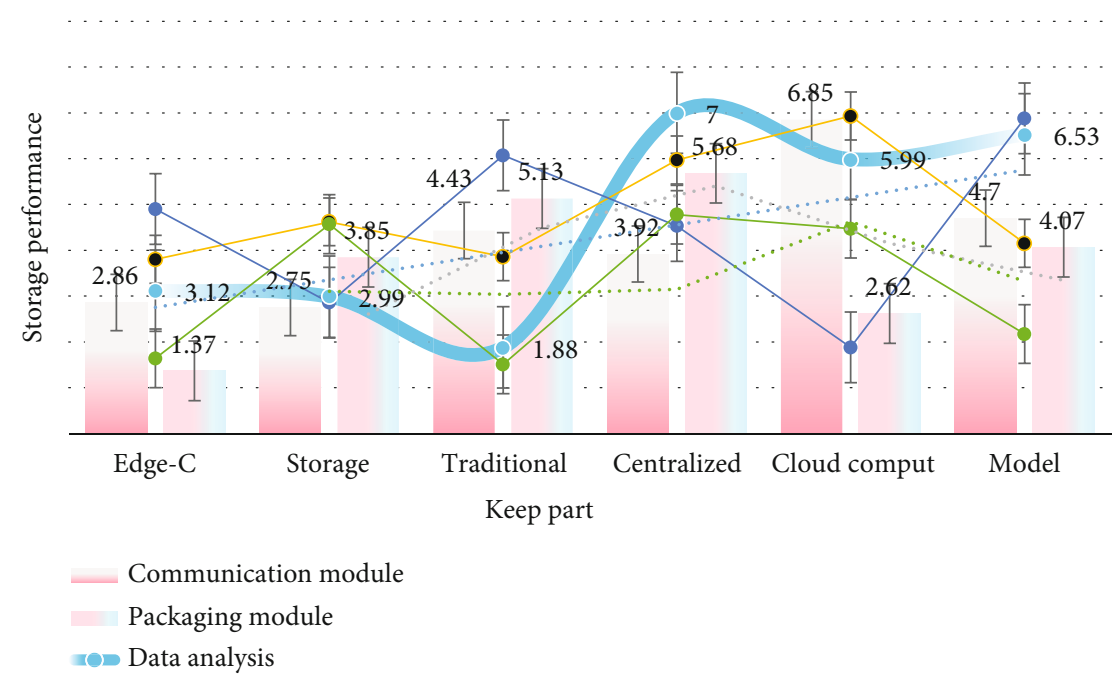

FIgURE 9: Computing layer close to the sensing layer device.

TABLE 7: Communication module provides device awareness for edge servers.

\begin{tabular}{lcccccc}
\hline & Communication module & Data analysis & Packaging module & Data calculation & Response module & Data storage \\
\hline Edge computing & 2.86 & 3.12 & 1.37 & 3.8 & 4.9 & 1.64 \\
Storage & 2.75 & 2.99 & 3.85 & 4.62 & 2.86 & 4.57 \\
Traditional & 4.43 & 1.88 & 5.13 & 3.86 & 6.07 & 1.51 \\
Centralized & 3.92 & 7 & 5.68 & 5.97 & 4.53 & 4.78 \\
Cloud computing & 6.85 & 5.99 & 2.62 & 6.93 & 4.88 & 6.88 \\
Model & 4.7 & 6.53 & 4.07 & 4.15 & & 2.17 \\
\hline
\end{tabular}

Redis storage and time series database in turn and indicates the synchronization priority of the data. According to the priority of data synchronization, the data is synchronized to the cloud computing layer in real time or regularly through the communication layer, and the data storage server of the cloud computing layer is updated. As shown in Table 8, according to the results of data processing, it is judged whether it is necessary to control the corresponding IoT devices to perform corresponding operations to obtain operating performance data.

The IoT devices in this system are mainly smart controllers and smart meters connected in series in the power circuit, as shown in Figure 12, deployed in each exhibition hall. Due to the deployment of more wireless access points on the exhibition hall floor, the campus network covers a wide range, and the signal is strong; smart controller devices use wireless communication protocols for communication, while smart meters use more stable wired communication. The most important equipment behavior of the intelligent controller is the on-off control of the power circuit and the power safety protection. The intelligent controller provides 10 branch circuit channels, which means that after the main circuit flows into the intelligent controller, multiple branch circuits can be separated, and through internal relays and control algorithms, different branch circuits can be controlled at the same time.

The smart controller replaces the traditional singlefunction wall switch. The user can control the on-off of the channel through the panel channel buttons of the smart controller and the corresponding APP, as shown in Table 9. The 


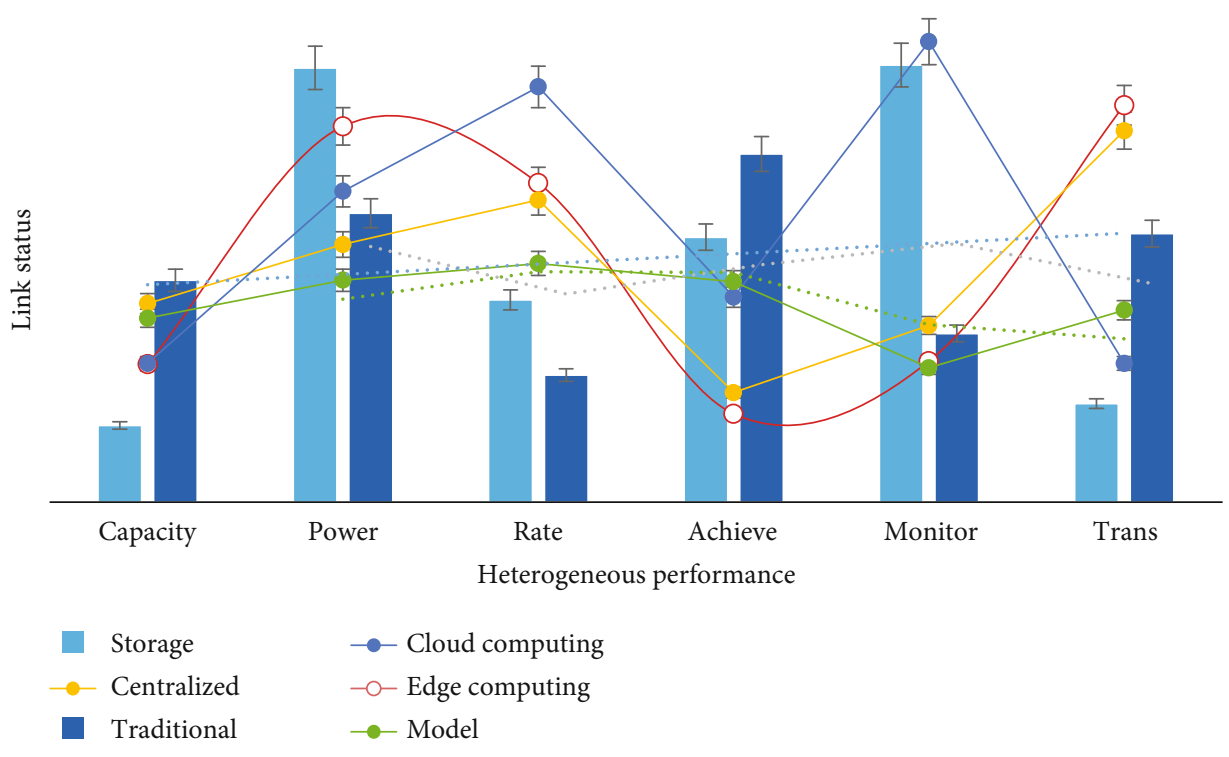

Figure 10: Perceive IoT devices and their communication status.

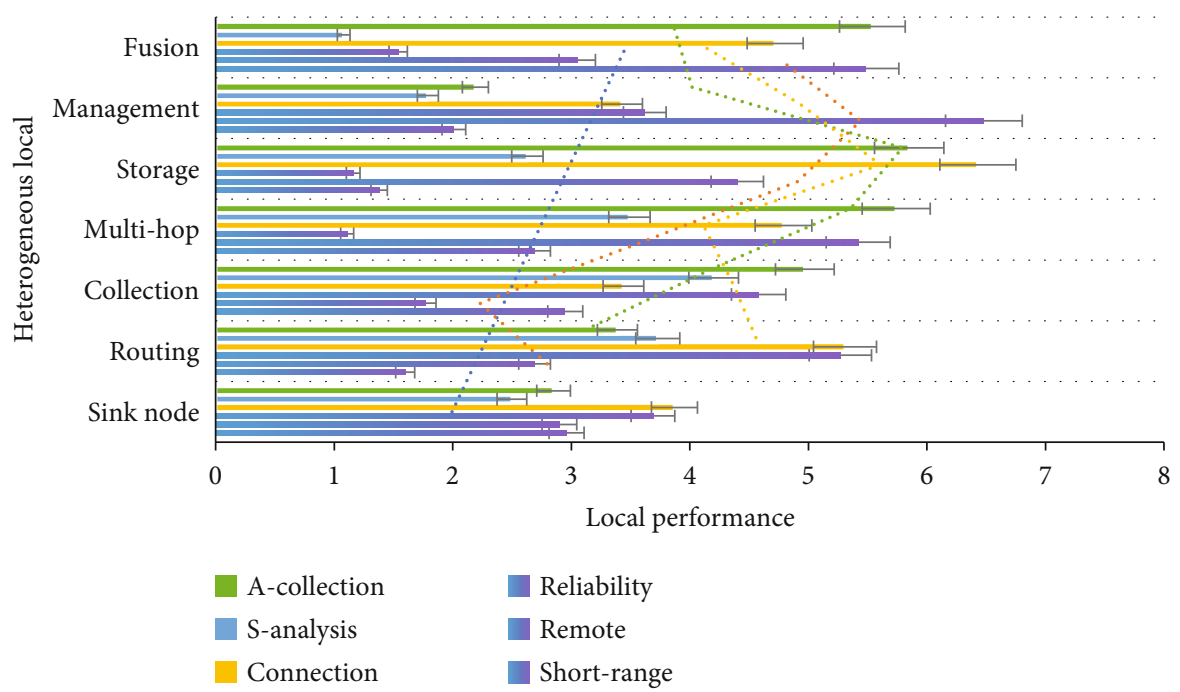

Figure 11: The communication layer synchronizes data to the cloud computing layer.

TABLE 8: Device control channel through cloud computing.

\begin{tabular}{|c|c|c|c|c|c|c|}
\hline Item & Short range & Remote & Reliability & Connection & S-analysis & A-collection \\
\hline Sink node & 2.96 & 2.9 & 3.69 & 3.87 & 2.5 & 2.85 \\
\hline Routing & 1.6 & 2.69 & 5.27 & 5.31 & 3.73 & 3.39 \\
\hline Collection & 2.95 & 1.77 & 4.58 & 3.44 & 4.2 & 4.97 \\
\hline Multihop & 2.69 & 5.42 & 1.11 & 4.79 & 3.49 & 5.74 \\
\hline Storage & 1.38 & 4.4 & 1.16 & 6.43 & 2.63 & 5.85 \\
\hline Management & 2.01 & 6.48 & 3.62 & 3.43 & 1.79 & 2.19 \\
\hline Fusion & 5.49 & 3.05 & 1.54 & 4.72 & 1.08 & 5.54 \\
\hline
\end{tabular}

system uses the first 6 channels of the intelligent controller, of which the first three channels are lighting channels, the fourth channel is socket channel, and the fifth and sixth channels are spare channels. Figure 8 shows the usage of each channel in Hall 1101 within one month. Among them, the use of the first three channels of the intelligent controller accounted for $79 \%$ of the daily use, and its use is greatly related to the life of the visitors. The types of exhibits in this system include graphic design, painting design, fashion design, industrial design, film and television animation, 


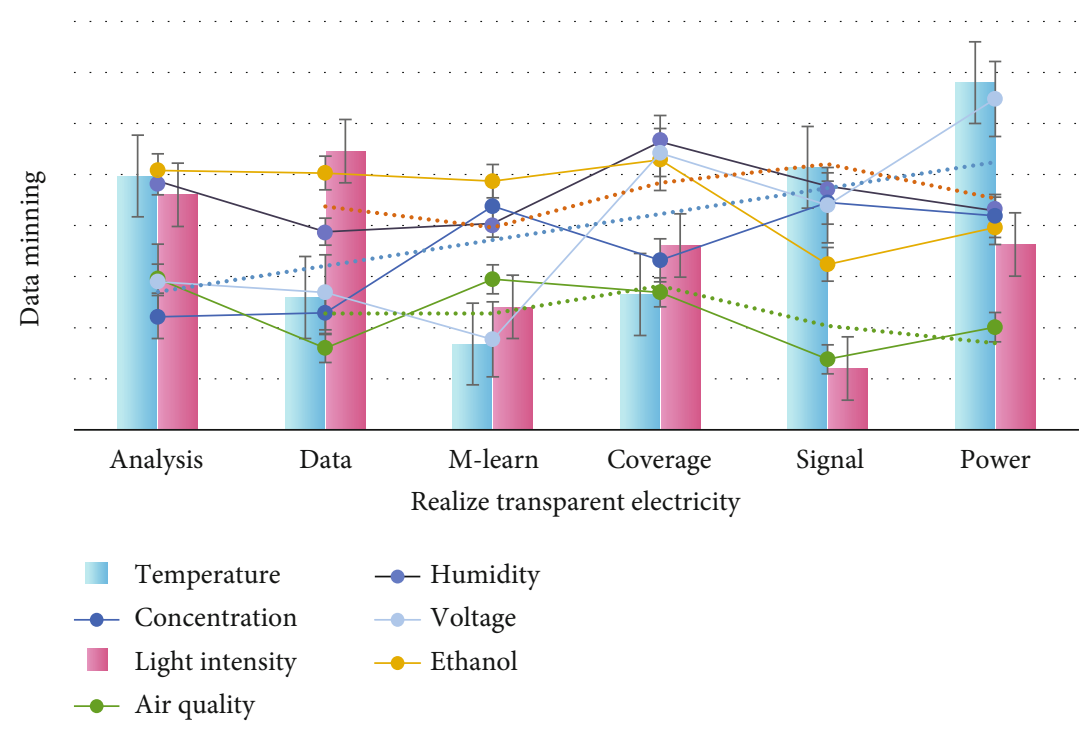

Figure 12: Smart controllers and smart meters in the loop.

TABle 9: Panel channel buttons of smart controller.

\begin{tabular}{|c|c|c|c|c|c|c|}
\hline Item & Short range & Remote & Reliability & Connection & S-analysis & A-collection \\
\hline Sink node & 5.31 & 3.73 & 3.39 & 5.03 & 2.68 & 2.08 \\
\hline Routing & 3.44 & 4.2 & 4.97 & 3.14 & 4.78 & 2.87 \\
\hline Collection & 4.79 & 3.49 & 5.74 & 1.42 & 2.46 & 1.78 \\
\hline Multihop & 6.43 & 2.63 & 5.85 & 4.9 & 2.6 & 3.22 \\
\hline Storage & 3.43 & 1.79 & 2.19 & 3.02 & 1.64 & 2.33 \\
\hline Management & 4.72 & 1.08 & 5.54 & 4.77 & 3.37 & 4.37 \\
\hline Fusion & 2.95 & 6.13 & 2.49 & 1.08 & 2.09 & 6.3 \\
\hline
\end{tabular}

architectural environment, art synthesis, digital photography, graduation design, and other types; exhibit formats include four types: video, audio, picture, and text. The management of the exhibits in this article can be realized from various presentation layer pages and back-end java source files. According to different management directions and user rights, under the premise of unified art, the front-end output of different types and formats of exhibits can be realized.

The main equipment behaviors of smart meters are electrical energy measurement and monitoring of voltage, current, and power. Through the measurement of the electric energy value, this article calculates the electricity consumption of the exhibition hall per hour, and this figure is usually directly related to the exhibition hall electricity bill. The histogram of electricity consumption in an exhibition hall within 3 months is shown in Figure 13. The electricity consumption includes fluorescent lamps, long-arm spotlights, LED lamps, air conditioners, sockets, and temperature and humidity control, and data is obtained through smart meters. Through clear electricity consumption data, transparent electricity consumption is realized and users' awareness of energy saving is enhanced. These data are uploaded to the cloud server, and the corresponding user energy consumption model is established through data analysis, data mining, machine learning, and other technologies, and the corresponding energy saving suggestions are given.

In this system, this article uses two indicators of communication round-trip delay and communication jitter to compare the traditional cloud computing system with this system. Among them, this article uses average deviation to define communication jitter, as shown in Table 10.

Figure 14 shows the result of the communication roundtrip delay between the traditional cloud computing system and this system. In traditional cloud computing systems, the communication round-trip delay time of the intelligent controller is mainly distributed between $80 \mathrm{~ms}$ and $260 \mathrm{~ms}$, with an average delay of $350 \mathrm{~ms}$. In the "direct connection" mode of this system, the communication round-trip delay time is mainly distributed between $65 \mathrm{~ms}$ and $80 \mathrm{~ms}$, and the average delay is $73.5 \mathrm{~ms}$. Through the calculation of the experimental results, it can be known that in the traditional cloud computing system, the communication jitter is $290 \mathrm{~ms}$, while in this system, the communication jitter is $16 \mathrm{~ms}$, which is about $1 / 4 \sim 1 / 5$ of the main communication delay interval. Based on the experimental results, it can be seen that the real-time performance of this system is far superior to the traditional cloud computing systems. The online display system of the artworks facilitates the college's 


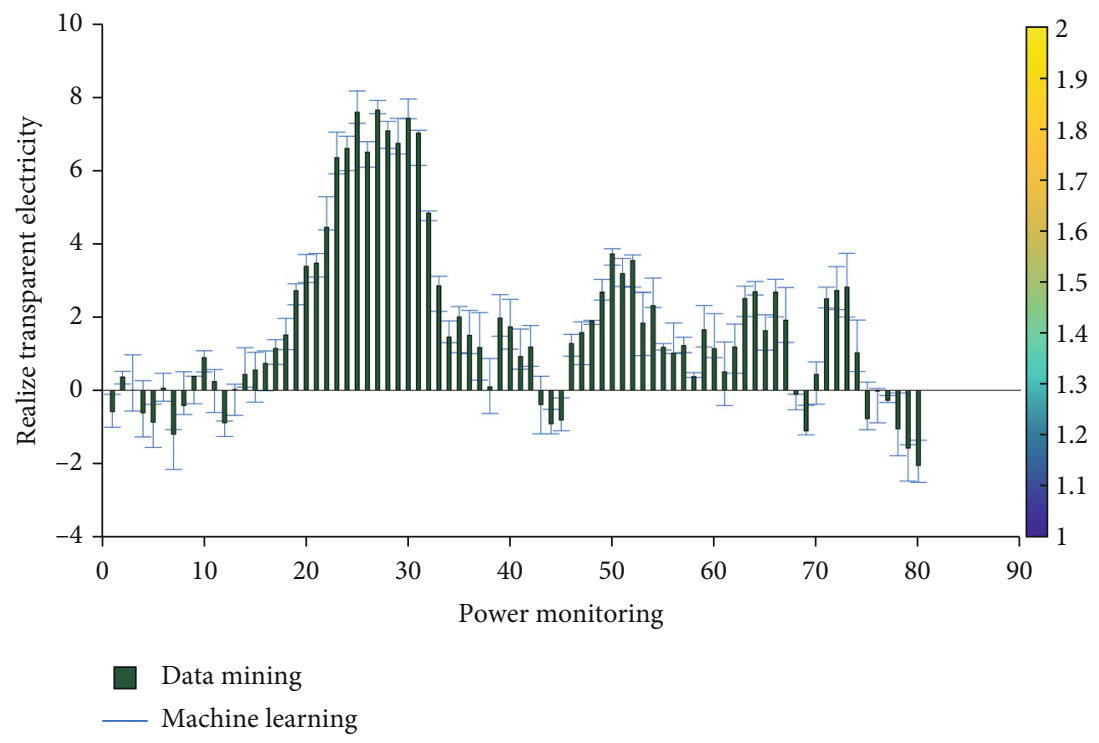

FIgURE 13: Electricity consumption of exhibition hall per hour.

Table 10: Mean deviation to define communication jitter.

\begin{tabular}{lccccccc}
\hline Item & Temperature & Humidity & Light intensity & Ethanol & Concentration & Air quality & Voltage \\
\hline Analysis & 4.97 & 4.87 & 4.6 & 5.08 & 2.21 & 2.96 & 2.9 \\
Data & 2.59 & 3.88 & 5.46 & 5.03 & 2.29 & 2.69 \\
M-learn & 1.68 & 4.04 & 2.41 & 4.87 & 4.38 & 1.6 & 2.95 \\
Coverage & 2.65 & 5.64 & 3.61 & 5.29 & 3.32 & 2.69 \\
Signa & 5.14 & 4.77 & 3.2 & 3.24 & 4.45 & 5.42 \\
Power & 6.8 & 4.29 & 3.63 & 3.96 & 4.19 & 4.38 \\
\hline
\end{tabular}

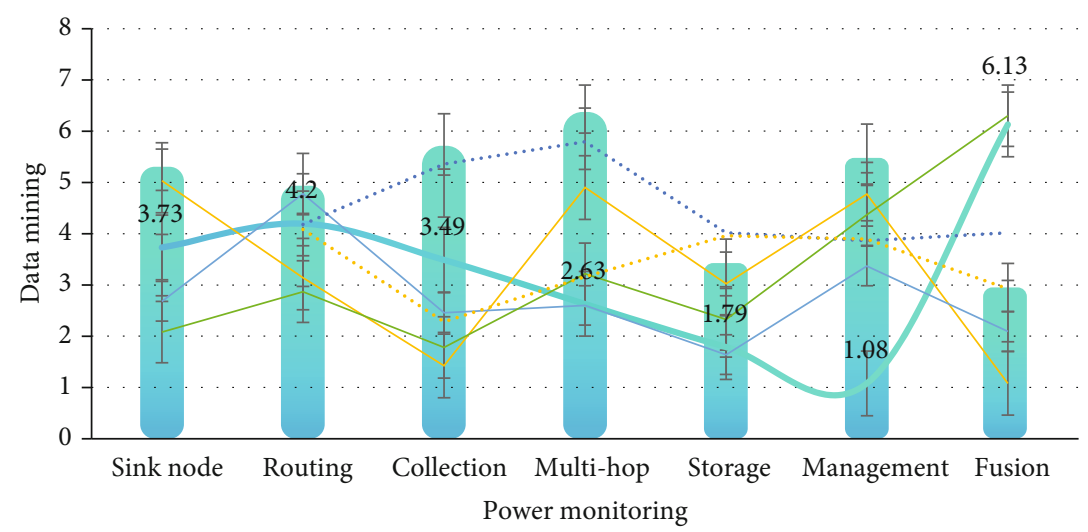

Short-range

Reliability

Remote

FIgure 14: Traditional cloud computing system and the system.

management of exhibits, exhibitions, and topics; facilitates users' inquiries and statistical analysis; standardizes the review process of exhibits; improves the quality and efficiency of exhibit information management; and is in line with the online exhibit management function, a practical online work information management platform. After the system is perfected, it will run successfully on the Internet platform. The system has intuitive interface and simple and easy-to-use functions, reduces a lot of manpower and time for exhibit management, and enhances efficiency. 


\section{Conclusions}

The equipment model of the artwork display system is a collection of equipment behaviors and an abstraction of IoT equipment. Usually, a device model contains multiple device behaviors. In the artwork display system, because the communication protocols of the connected perception layer devices are different, the same device behavior often has many different expressions under different device models, which is easy to cause errors. The system converts the device information in the form of "many-to-one" to unify the data format, facilitate server data processing, and write the conversion relationship into the system configuration table. When the data is sent from the edge server to the perception layer device, the data can be correctly distributed through the conversion relationship for data packaging. As long as the conversion relationship is filled in correctly, the system does not need to pay attention to the heterogeneity among IoT devices, reducing the difficulty of system development.

When data is sent from the edge layer to the cloud computing layer, Google's open source protocolbuffer protocol (protolbuf for short) is used to encapsulate the data. The protolbuf protocol has a certain encryption capability for information, and when the amount of information is constant, the information transmission efficiency is higher and the communication flow is reduced. The data processing and response module is the core part of the edge server. This module determines the priority of data processing by identifying the source and type of data. For high-priority data, it often seizes the server's computing resources, obtains the results, and executes the tasks in accordance with the established procedures to improve the real-time performance of the system. Different from cloud computing storage, due to limited storage resources, edge servers only include Redis storage and time series data databases.

This article introduces the design of the artwork display system in detail, focusing on the use of the knowledge of the Internet of Things, but it can be found that there are many deficiencies in the article, mainly reflected in the inadequacy of data processing. In response to this problem, in the follow-up research, in-depth analysis of big data processing technology will be carried out. Combined with the Internet of Things technology, the system can be made more intelligent and more powerful.

\section{Data Availability}

The data that support the findings of this study are available from the corresponding author upon reasonable request.

\section{Conflicts of Interest}

The authors declare that they have no conflicts of interest.

\section{References}

[1] J. H. Zhu, J. Wu, and Y. Tao, "Sensor optimal placement algorithm based on coverage rate," Computer Engineering, vol. 36, no. 3, pp. 94-96, 2020.
[2] L. Levin, M. Segal, and H. Shpungin, "Cooperative data collection in ad hoc networks," Wireless Networks, vol. 19, no. 2, pp. 145-159, 2013.

[3] S. Chakraborty, S. Chakraborty, S. Nandi, and S. Karmakar, "ADCROSS: adaptive data collection from road surveilling sensors," IEEE Transactions on Intelligent Transportation Systems, vol. 15, no. 5, pp. 2049-2062, 2014.

[4] C. Zou, W. Jiang, and F. Tsung, "A LASSO-based diagnostic framework for multivariate statistical process control," Technometrics, vol. 53, no. 3, pp. 297-309, 2011.

[5] C. Zou, Z. Wang, X. Zi, and W. Jiang, "An efficient online monitoring method for high-dimensional data streams," Technometrics, vol. 57, no. 3, pp. 374-387, 2019.

[6] Q. Wei, W. Huang, W. Jiang, and W. Zhao, "Real-time process monitoring using kernel distances," International Journal of Production Research, vol. 54, no. 21, pp. 6563-6578, 2016.

[7] L. E. Jian, L. I. U. Yongyan, Y. E. Xi, Z. H. O. U. Wu, and L. I. Zhiwei, "Market-oriented operation pattern of regional power network integration with high penetration level of distributed energy resources," Proceedings of the CSEE, vol. 36, no. 12, pp. 3343-3354, 2016.

[8] L. I. Rui, "Comprehensive benefit evaluation method of distributed generation microgrid projects based on different business models," Power System Technology, vol. 41, no. 6, pp. 1748-1758, 2017.

[9] W. A. N. G. Xuanyuan, L. I. U. Dunnan, and L. I. U. Zhen, "Operation mechanism and key technologies of virtual power plant under ubiquitous IoT," Power System Technology, vol. 43, no. 9, pp. 3175-3183, 2019.

[10] L. I. U. Riliang, L. I. U. Haitao, and X. I. A. Shengfeng, "IoT technology application and prospects in distribution transformer service area management," High $V$ oltage Engineering, vol. 45, no. 6, pp. 1707-1714, 2019.

[11] R. S. Sinha, Y. Wei, and S.-H. Hwang, "A survey on LPWA technology: LoRa and NB-IoT," ICT Express, vol. 3, no. 1, pp. 14-21, 2017.

[12] Y.-P. E. Wang, X. Lin, A. Adhikary et al., "A primer on 3GPP narrowband Internet of Things," IEEE Communications Magazine, vol. 55, no. 3, pp. 117-123, 2017.

[13] F. Liang, L. H. Yin, and Y. C. Guo, "A survey of key technologies in attribute-based access control scheme," Chinese Journal of Computers, vol. 40, no. 7, pp. 1680-1698, 2017.

[14] A. Ouaddah, A. Abou Elkalam, and O. A. Ait, "FairAccess: a new blockchain-based access control framework for the Internet of Things," Security \& Communication Networks, vol. 9, no. 18, pp. 5943-5964, 2016.

[15] A. Ouaddah, A. A. Elkalam, and A. A. Ouahman, "Towards a novel privacy-preserving access control model based on blockchain technology in IoT," in Europe, Middle East and North Africa Conference on Technology and Security to Support Learning, EMENA-TSSL 2016, vol. 5no. 6, pp. 523-533, Saidia, Oujda, Morocco: Springer Verlag, 2017.

[16] A. Outchakoucht, H. Es-Samaali, and J. Philippe, "Dynamic access control policy based on blockchain and machine learning for the IoT," International Journal of Advanced Computer ence and Applications, vol. 8, no. 7, pp. 417424, 2017.

[17] D. D. F. Maesa, P. Mori, and L. Ricci, "Blockchain based access control," in IFIP international conference on distributed applications and interoperable systems, vol. 8no. 5, pp. 206-220, Neuchâtel, Switzer-land: Springer, Cham, 2017. 
[18] S. Ding, J. Cao, C. Li, K. Fan, and H. Li, "A novel attributebased access control scheme using blockchain for IoT," IEEE Access, vol. 7, no. 45, pp. 38431-38441, 2019.

[19] J. S. Shi and R. Li, "Survey of blockchain access control in IoT," Journal of Software, vol. 30, no. 6, pp. 1632-1648, 2019.

[20] A. Z. Ourad, B. Belgacem, and K. Salah, "Using blockchain for IoT access control and authentication management," in International Conference on IoT, vol. 7no. 6, pp. 150-164, Seattle, WA, United states: Springer Verlag, 2018.

[21] I. Riabi, Y. Dhif, and H. K. B. Ayed, "A blockchain based access control for IoT," in 2019 15th international wireless communications and Mobile computing conference (IWCMC), vol. 7no. 32, pp. 2086-2091, Tangier, Morocco: Institute of Electrical and Electronics Engineers Inc, 2019.

[22] K. Koštál, P. Helebrandt, M. Belluš, M. Ries, and I. Kotuliak, "Management and monitoring of IoT devices using blockchain," Sensors, vol. 19, no. 4, pp. 856-868, 2019.

[23] E. Adi, A. Anwar, Z. Baig, and S. Zeadally, "Machine learning and data analytics for the IoT," Neural Computing and Applications, vol. 32, no. 20, pp. 16205-16233, 2020.

[24] H. Gao, Z. Ma, S. Luo, Y. Xu, and W. Zheng, "BFR-SE: a blockchain-based fair and reliable searchable encryption scheme for IoT with fine-grained access control in cloud environment," Wireless Communications and Mobile Computing, vol. 2021, 21 pages, 2021.

[25] Z. H. A. N. G. Liang, L. I. U. Baixiang, Z. H. A. N. G. Ruyi, J. I. A. N. G. Binxin, and L. I. U. Yijiang, "Overview of blockchain technology," Computer Engineering, vol. 45, no. 5, pp. 1-12, 2019. 